\title{
The variable XMM-Newton spectrum of Markarian 766^
}

\author{
M. J. Page ${ }^{1}$, K. O. Mason ${ }^{1}$, F. J. Carrera ${ }^{2}$, J. Clavel $^{3}$, J. S. Kaastra ${ }^{4}$, E. M. Puchnarewicz ${ }^{1}$, \\ M. Santos-Lleo ${ }^{3}$, H. Brunner ${ }^{5}$, C. Ferrigno ${ }^{4}$, I. M. George ${ }^{6}$, F. Paerels ${ }^{4}$, K. A. Pounds ${ }^{7}$, \\ and S. P. Trudolyubov ${ }^{8}$
}

1 Mullard Space Science Laboratory, University College London, Holmbury St Mary, Dorking, Surrey, RH5 6NT, UK

2 Instituto de Física de Cantabria (Consejo Superior de Investigaciones Científicas-Universidad de Cantabria), 39005 Santander, Spain

3 XMM-Newton Science Operations Centre, Astrophysics Division, ESA Space Science Department, PO Box 50727, 28080 Madrid, Spain

4 Space Research Organization of The Netherlands, Laboratory for Space Research, Sorbonnelaan 2, Utrecht, CA 3584, The Netherlands

5 Astrophysikalisches Institut Potsdam, Sternwarte 16, 14482 Potsdam, Germany

6 NASA Goddard Space Flight Center, Code 660, Greenbelt, MD 20771, USA

7 X-Ray Astronomy Group, Department of Physics and Astronomy, Leicester University, Leicester LE1 7RH, UK

8 Los Alamos National Laboratory, NIS-2, Los Alamos, NM 87545, USA

Received 2 October 2000 / Accepted 10 November 2000

\begin{abstract}
The narrow-line Seyfert 1 galaxy Mrk 766 was observed for 60 ks with the XMM-Newton observatory. The source shows a complex X-ray spectrum. The $2-10 \mathrm{keV}$ spectrum can be adequately represented by a power law and broad Fe $\mathrm{K} \alpha$ emission. Between 0.7 and $2 \mathrm{keV}$ the spectrum is harder and exhibits a flux deficit with respect to the extrapolated medium energy slope. Below $0.7 \mathrm{keV}$, however, there is a strong excess of emission. The RGS spectrum shows an edge-like feature at $0.7 \mathrm{keV}$; the energy of this feature is inconsistent with that expected for an OVII edge from a warm absorber. Mrk 766 varies by a factor of $\sim 2$ in overall count rate in the EPIC and RGS instruments on a timescale of a few thousand seconds, while no significant flux changes are observed in the ultraviolet with the OM. The X-ray variability is spectrally dependent with the largest amplitude variability occurring in the $0.4-2 \mathrm{keV}$ band. The spectral variability can be explained by a change in flux and slope of the medium energy continuum emission, superimposed on a less variable (or constant) low energy emission component.
\end{abstract}

Key words. accretion, accretion disks - black hole physics - galaxies: Seyfert - X-rays: general

\section{Introduction}

X-ray emission currently provides the best tracer of conditions close to the supermassive black hole central engines of AGN. However the origin of AGN X-ray emission remains poorly understood. Between 1 and $20 \mathrm{keV}$ AGN generally have a power law spectrum modified by the effects of reflection and absorption (Pounds et al. 1990); below 1 keV many AGN also show some "soft excess" emission (Walter \& Fink 1993). The majority of recent models for AGN X-ray emission explain the soft excess as

Send offprint requests to: M. J. Page,

e-mail: mjp@mssl.ucl.ac.uk

* Based on observations obtained with XMM-Newton, an ESA science mission with instruments and contributions directly funded by ESA Member States and the USA (NASA). emission from an optically thick accretion disk, and the power law component as Compton upscattering of soft photons by a hot corona.

Mrk 766 is a nearby $(z=0.0129)$, soft X-ray bright $\left(0.5-2 \mathrm{keV}\right.$ flux of $\sim 10^{-11} \mathrm{erg} \mathrm{s}^{-1} \mathrm{~cm}^{-2}$ ) and variable (factor 2 in $\sim 1000 \mathrm{~s}$, Leighly et al. 1996) narrow line Seyfert 1 galaxy observed through a relatively small Galactic column of $\sim 1.810^{20} \mathrm{~cm}^{-2}$. It is therefore an extremely good candidate for studying the dynamical and radiative processes which give rise to the X-ray emission in AGN. In this letter we present the spectrum and spectral variability of Mrk 766 as observed by the combination of instruments on board XMM-Newton during the performance verification (PV) observation. 


\section{The XXM-Newton observation and data reduction}

The observation was performed on 20th May 2000 during $X M M-N e w t o n$ revolution 0082 and lasted $\sim 60 \mathrm{ks}$. All instruments suffered some data gaps close to the end of the observation. The data were processed using the standard analysis software (SAS) pipeline. For EPIC MOS1 and $\mathrm{PN}$ analysis we use the most up to date (October 2000) on-axis response matrices available. The RGS data were analysed using response matrices generated by the SAS.

Due to high particle background near the beginning of the observation, the EPIC PN and MOS cameras were closed for the first $20 \mathrm{ks}$ and $30 \mathrm{ks}$ respectively. The EPIC MOS cameras were operated in full frame imaging mode. The observed countrate of Mrk 766 is about five times larger than the recommended limit for this imaging mode, and therefore the MOS data are piled up. This is expected to result in a hardening of the MOS spectrum equivalent to a change in power law energy index of $\Delta \alpha \sim 0.05$ (Fig. 28 in the XMM-Newton User Handbook). Apart from this spectral hardening, we do not expect photon pile-up to seriously degrade the spectra. MOS spectra used in this paper were constructed using pattern 0-12 events. Source data were taken from a forty arcsecond radius region around the source, and background data were taken from four identical circular regions close to the source position. MOS Spectra were grouped to a minimum of 150 counts per group.

The EPIC PN camera was used in small window mode and is not significantly piled-up. Source data were extracted from a 35 arcsec radius region and background data were taken from three identical circular regions. Only single pixel (pattern 0) events were used to construct PN spectra, to match the available response matrix. In order to overcome a problem in current versions of the SAS, which produces periodic dips every $\sim 25 \mathrm{eV}$ in PN spectra, we have manually grouped $\mathrm{PN}$ spectra to groups which are $>25 \mathrm{eV}$. Less than $1 \%$ of the counts in the source regions in the MOS and PN cameras are due to the background.

Both RGS cameras were operated in the standard spectroscopy $+\mathrm{Q}$ mode for the duration of the observation. Source data were extracted using the standard spatial and order selections, and background data were taken from two identical sized extraction regions offset from the source in the cross dispersion direction. $87 \%$ of the counts in the source regions come from Mrk 766 .

The OM took a sequence of 5 exposures through the UVW1 filter, 10 exposures through the UVM2 filter, and 3 exposures through the UVW2 filter.

\section{The spectra}

The EPIC PN, and MOS spectra of Mrk 766 are shown in Fig. 1a, and were analysed using the XSPEC software package.

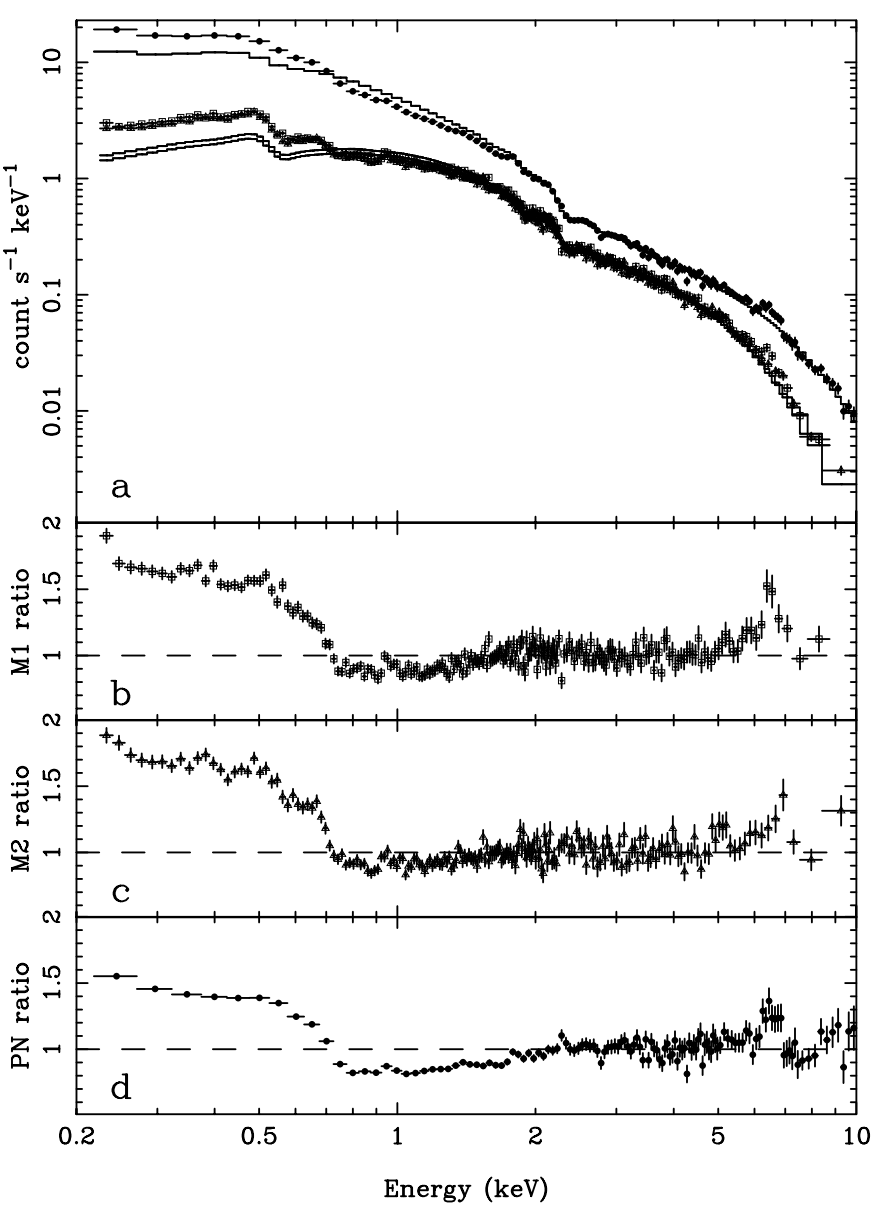

Fig. 1. a) Spectra from MOS1 (open squares), MOS2 (open triangles), PN (filled dots) and power laws convolved with the instrument response functions (stepped lines). b-d) Ratios of MOS1, MOS2, and PN spectra to the power law model

Table 1. Power law $\left(F_{\mathrm{E}}=K E^{-\alpha}\right)$ fits to the EPIC spectra in the $2-5$ and $7-10 \mathrm{keV}$ energy ranges

\begin{tabular}{lccc}
\hline Instrument & $\alpha$ & $K^{*}$ & $\chi^{2} / \nu$ \\
\hline $\mathrm{M} 1$ & $0.98 \pm 0.03$ & $4.66 \pm 0.16$ & $66.5 / 76$ \\
$\mathrm{M} 2$ & $0.96 \pm 0.03$ & $4.30 \pm 0.16$ & $87.4 / 72$ \\
$\mathrm{PN}$ & $1.09 \pm 0.02$ & $6.11 \pm 0.15$ & $71.8 / 71$ \\
PN high & $1.14 \pm 0.03$ & $7.36 \pm 0.23$ & $12.7 / 18$ \\
PN low & $1.05 \pm 0.03$ & $5.21 \pm 0.18$ & $19.0 / 18$ \\
\hline${ }^{*} 10^{-3} \mathrm{keV} \mathrm{cm}^{-2} \mathrm{~s}^{-1} \mathrm{keV}^{-1}$. &
\end{tabular}

\section{1. $2-10 \mathrm{keV}$ spectrum}

We began studying the spectra by fitting a power law model $\left(F_{\mathrm{E}} \propto E^{-\alpha}\right)$ with a Galactic $N_{\mathrm{H}}$ of $1.810^{20} \mathrm{~cm}^{-2}$ to the EPIC spectra in the energy ranges $2-5$ and $7-10 \mathrm{keV}$ where the spectrum should be relatively unaffected by warm absorbers, Fe line emission or a soft excess. Table 1 shows the results of these fits. The data in these energy ranges are indeed fit well by a power law model. The best fit slopes for the MOS data are harder than those for the 
Table 2. Fe K $\alpha$ line parameters when the $2-10 \mathrm{keV}$ spectrum is fit with a power law + Gaussian emission line

\begin{tabular}{lcccc}
\hline Instrument & Energy $^{*}$ & $\sigma^{*}$ & $E W^{*}$ & $\chi^{2} / \nu$ \\
\hline M1 & $6.5 \pm 0.2$ & $0.44_{-0.16}^{+0.20}$ & $0.38_{-0.08}^{+0.20}$ & $78.2 / 88$ \\
M2 & $6.8_{-0.5}^{+0.2}$ & $0.39_{-0.21}^{+0.49}$ & $0.26_{-0.16}^{+0.20}$ & $110 / 83$ \\
PN & $6.5 \pm 0.1$ & $0.24_{-0.05}^{+0.05}$ & $0.19_{-0.03}^{+0.04}$ & $90.4 / 89$ \\
\hline
\end{tabular}

${ }^{*}$ Rest frame in units of $\mathrm{keV} . E W$ is equivalent width.

PN data by $\Delta \alpha \sim 0.1$; this is probably due to pile-up in the MOS cameras (see Sect. 2). The data and model (including the $0.2-2$ and $5-7 \mathrm{keV}$ regions not used in the fit) are shown in Fig. 1a. The ratio of the data to the model is shown for the three EPIC cameras in Figs. 1b,c, and d.

A prominent feature, consistent with a broad Fe $\mathrm{K} \alpha$ emission line centred at $6.4 \mathrm{keV}$, is present in the spectra from all 3 EPIC cameras. An emission line component is strongly required by the data: fitting the whole $2-10 \mathrm{keV}$ PN spectrum with only a power law and fixed Galactic $N_{\mathrm{H}}$ results in a $\chi^{2} / \nu$ of $127 / 92$, unacceptable at $>99 \%$ confidence. The presence of $\mathrm{Fe} \mathrm{K} \alpha$ emission in Mrk 766 has so far been controversial: Leighy et al. (1996) found evidence for a narrow $\mathrm{Fe} \mathrm{K} \alpha$ line, Nandra 1997 found evidence for a broad $\mathrm{Fe} \mathrm{K} \alpha$ line (both using $A S C A$ data), while Matt et al. (2000) found no strong evidence for an $\mathrm{Fe}$ $\mathrm{K} \alpha$ line at all in their BeppoSAX data. The results of fits to the $2-10 \mathrm{keV}$ spectrum with a power law continuum and Gaussian emission line model are given in Table 2, and residuals to these fits are shown in Fig. 2. The Fe $\mathrm{K} \alpha$ line in Seyfert 1 galaxies is widely thought to arise from fluorescence in near neutral material in an accretion disk around a black hole. An emission line produced by this process typically has an asymmetric profile with significant broadening to the red. The residuals to the Gaussian line fits (Fig. 2) show an excess of emission between 5 and $5.5 \mathrm{keV}$, suggesting that the emission line in Mrk 766 has such a red wing. We have therefore investigated accretion disk emission line models. Table 3 gives the results of model fits to the $\mathrm{Fe} \mathrm{K} \alpha$ line in the $2-10 \mathrm{keV} \mathrm{PN}$ data, which have the highest signal to noise. This time we have used emission line profiles appropriate for accretion disks around a maximally rotating Kerr black hole (Laor 1991, the LAOR model in XSPEC) and a Schwarzschild black hole (Fabian et al. 1989, the DISKLINE model in XSPEC). Both models fit the data well, and therefore the data are consistent with the $\mathrm{Fe} \mathrm{K} \alpha$ line originating in an accretion disk. There is little to distinguish between the Kerr and Schwarzschild models in terms of $\chi^{2} / \nu$.

Matt et al. (2000) found that fits to their BeppoSAX spectra of Mrk 766 were significantly improved with the addition of an edge at $7.55 \mathrm{keV}$, or an ionized reflection component (which produces an edge at a similar energy). We have repeated fits to our $2-10 \mathrm{keV}$ spectra with power law and emission line models, adding either an edge at $\sim 7.5 \mathrm{keV}$, or an ionized reflector (the PEXRIV model in XSPEC). For all three EPIC spectra, and for both

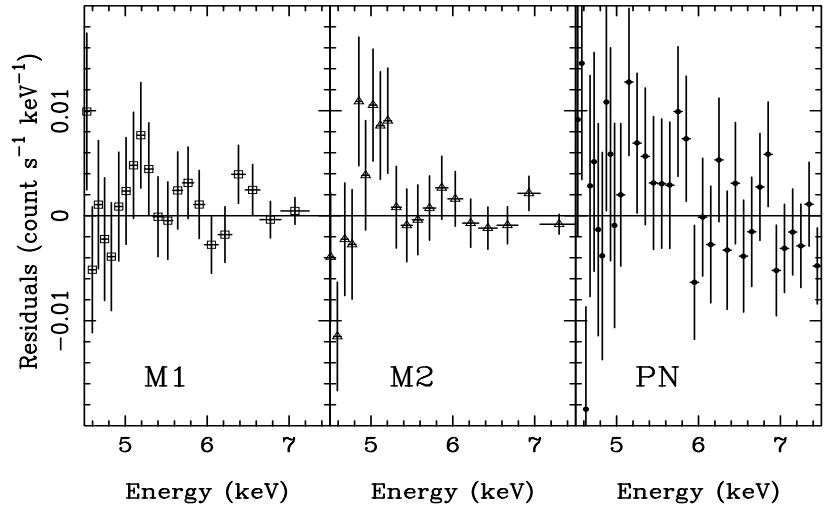

Fig. 2. Residuals of the Gaussian fits to the $\mathrm{Fe} \mathrm{K} \alpha$ line (Table 2). Note the excess emission at $5-5.5 \mathrm{keV}$ indicating that the emission line has a red wing

Table 3. Relativistic accretion disk fits to the $\mathrm{Fe} \mathrm{K} \alpha$ line in the PN data

\begin{tabular}{lll}
\hline Model & Schwarzschild & Kerr \\
\hline Energy $^{a}$ & $6.5 \pm 0.1$ & $6.6 \pm 0.1$ \\
$E W^{a}$ & $0.29 \pm 0.07$ & $0.37 \pm 0.11$ \\
Inclination & $34^{\circ} \pm 7^{\circ}$ & $30^{\circ} \pm 6^{\circ}$ \\
$q^{b}$ & $2.5_{-0.5}^{+1.6}$ & $3.2_{-0.4}^{+3.5}$ \\
$\chi^{2} / \nu$ & $86.3 / 88$ & $85.1 / 88$ \\
\hline
\end{tabular}

${ }^{a}$ Rest frame in units of $\mathrm{keV} . E W$ is equivalent width. ${ }^{b}$ power law dependence of emissivity on radius: emissivity $\propto$ $R^{-q}$. Inner disk radius fixed at $6 R_{\mathrm{g}}$.

additional components, improvements to the $\chi_{\nu}^{2}$ are less than $2 \sigma$ significant according to the F-test, and we therefore conclude that the spectra do not require an ionized reflection component.

\section{2. $0.2-2 \mathrm{keV}$ spectrum}

The EPIC data shown in Fig. 1 show that between 0.7 and $2 \mathrm{keV}$ the spectrum is harder than the power law extrapolation. Below $0.7 \mathrm{keV}$ a strong excess is seen. Figure 3 shows the spectrum of Mrk 766 recorded by the two RGS, in bins of 4 channels to improve signal to noise. The spectrum shows no strong narrow emission lines. This rules out a significant nuclear starburst contribution to the soft X-ray emission, which was suggested by Mathur (2000). The most notable feature in the spectrum is a discontinuity at $0.7 \mathrm{keV}$. This feature corresponds to the highest energy of the soft excess emission seen in the EPIC spectra. A similar feature was previously identified with the K-edge of OVII from a warm absorber in $A S C A$ and BeppoSAX data by Leighly et al. (1996) and Matt et al. (2000) respectively; the presence of an OVIII K-edge was also inferred from both these datasets. At the redshift of Mrk 766 (0.0129) the OVII edge would be found at $0.730 \mathrm{keV}$ and the OVIII edge at $0.860 \mathrm{keV}$; these energies are labelled in Fig. 3. Edges are not seen in the RGS data at either 


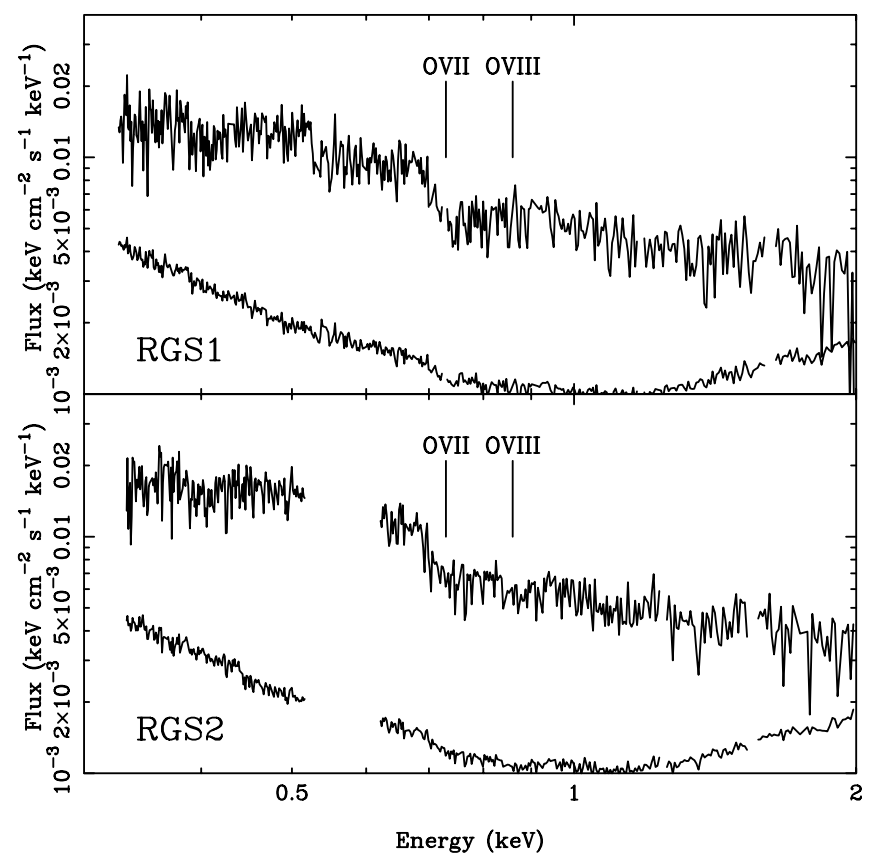

Fig. 3. Spectra of Mrk 766 recorded by RGS1 (top panel) and RGS2 (bottom panel). The lower line in each panel shows the size of the $1 \sigma$ statistical uncertainty. The positions of the expected warm absorber OVII and OVIII K-edges are labelled

of these energies. If the feature at $0.7 \mathrm{keV}$ is an OVII K-edge, then the absorbing material must be redshifted by more than $10000 \mathrm{~km} \mathrm{~s}^{-1}$ which is inconsistent with current physical scenarios for the warm absorber gas, e.g. that it is in an outflowing wind (Kaastra et al. 2000) or that it is associated with the broad and/or narrow line regions (Otani et al. 1996). If the deficit of photons between 0.7 and $2.0 \mathrm{keV}$ is not produced by an ionized absorber, the continuum itself must deviate from a power law shape, and have a harder spectrum in this energy range than at higher energies.

Detailed modelling of the soft X-ray spectrum of Mrk 766 in this energy range is outside the scope of this letter. For a more detailed examination of the discrepancy between the Mrk 766 RGS data and warm absorber models, see Branduardi-Raymont et al. (2001) who model the features in the RGS spectrum as emission lines from a relativistic accretion disk.

\section{Lightcurves}

RGS, EPIC PN and OM lightcurves of Mrk 766 are shown in Fig. 4. The EPIC MOS lightcurves (not shown) are similar to the PN lightcurves but cover a shorter timespan and have lower signal to noise. The RGS and PN lightcurves are constructed with $300 \mathrm{~s}$ bins. The PN lightcurves have been split into four different energy bands $(0.1-0.4 \mathrm{keV}$, 0.4-2.0 keV, 2.0-6.0 keV, and > $6.0 \mathrm{keV}$ ) and are shown in panels b-e of Fig. 4. Hardness ratios between the 0.1-0.4 keV, 0.4-2.0 keV and 2.0-6.0 keV energy bands are shown in panels $\mathrm{g}$ and $\mathrm{h}$ of Fig. 4. The low energy
$(0.4-2.0 \mathrm{keV}) /(0.1-0.4 \mathrm{keV})$ hardness ratio varies such that Mrk 766 is harder when it is brighter. This is suggestive of a scenario in which the variability of a broad band emission component is diluted at the softest energies because of the superposition of a relatively constant soft excess component. Although the correspondence is not exact, for most of the observation the $(2.0-6.0 \mathrm{keV}) /(0.4-$ $2.0 \mathrm{keV})$ hardness ratio appears to show the opposite behaviour to the lower energy hardness ratio: the $0.4-$ $6.0 \mathrm{keV}$ spectrum is harder when the $0.1-2.0 \mathrm{keV}$ spectrum is softer and vice versa.

We have quantified the variability in each of the PN lightcurves using the normalised variability amplitude (hereafter NVA) which we define as:

$\mathrm{NVA}=\frac{1}{\bar{x}} \sqrt{\left(\frac{1}{(N-1)} \sum_{i=1}^{N}\left(x_{i}-\bar{x}\right)^{2}\right)-\sigma^{2}}$

where $\sigma$ is the statistical uncertainty on the data points $x_{i}$. $1 \sigma$ statistical uncertainties on the NVAs were estimated by bootstrap resampling of the lightcurves. The NVAs of the $0.1-0.4 \mathrm{keV}, 0.4-2.0 \mathrm{keV}, 2.0-6.0 \mathrm{keV}$ and $>6.0 \mathrm{keV}$ lightcurves are $0.15 \pm 0.01,0.19 \pm 0.01,0.17 \pm 0.01$ and $0.12 \pm 0.02$ respectively. The $0.4-2.0 \mathrm{keV}$ lightcurve shows more variability than the low energy $(0.4-2.0)$ and high energy (>6.0 keV) lightcurves.

The longest OM sequence of exposures (UVM2) is shown in Fig. 4f. In the ultraviolet there is little evidence for variability: a constant is an acceptable fit to the UVM2 lightcurve $\left(\chi^{2} / \nu\right.$ of $\left.11.1 / 9\right)$ and the data are all within $7 \%$ of the mean countrate. Similarly, no variability is found in the shorter UVW1 and UVW2 sequences (not shown).

\section{Spectral variability}

To examine how the spectrum of Mrk 766 changes with flux, we have divided the observation into two parts corresponding to "high flux" and "low flux". These time periods are indicated in Fig. 4a. The EPIC PN spectra from the high and low periods are shown in Fig. 5a, after division by a model power law with energy index $\alpha=1.09$ (the best fit slope for the $2-10 \mathrm{keV}$ PN spectrum of the whole observation) and fixed Galactic $N_{\mathrm{H}}$. In order to improve signal to noise, the PN high and low flux spectra have been grouped more heavily than the PN spectrum for the whole observation. The main difference between the two spectra is that the overall continuum is higher in the higher flux spectrum.

The ratio of the high to low flux spectra is shown in Fig. 5b. This shows that between 1 and $10 \mathrm{keV}$ the high flux spectrum is softer than the low flux spectrum, while below $1 \mathrm{keV}$ the high flux spectrum is harder than the low flux spectrum. We have performed power law fits to the $\mathrm{PN}$ data in the $2-5$ and $7-10 \mathrm{keV}$ energy range for the high and low flux spectra separately, and the results of these fits are given in Table 1 . The best-fit power law to the high flux spectrum is softer than the best fit to the low flux spectrum by $\Delta \alpha=0.1$, significant at $2 \sigma$. 


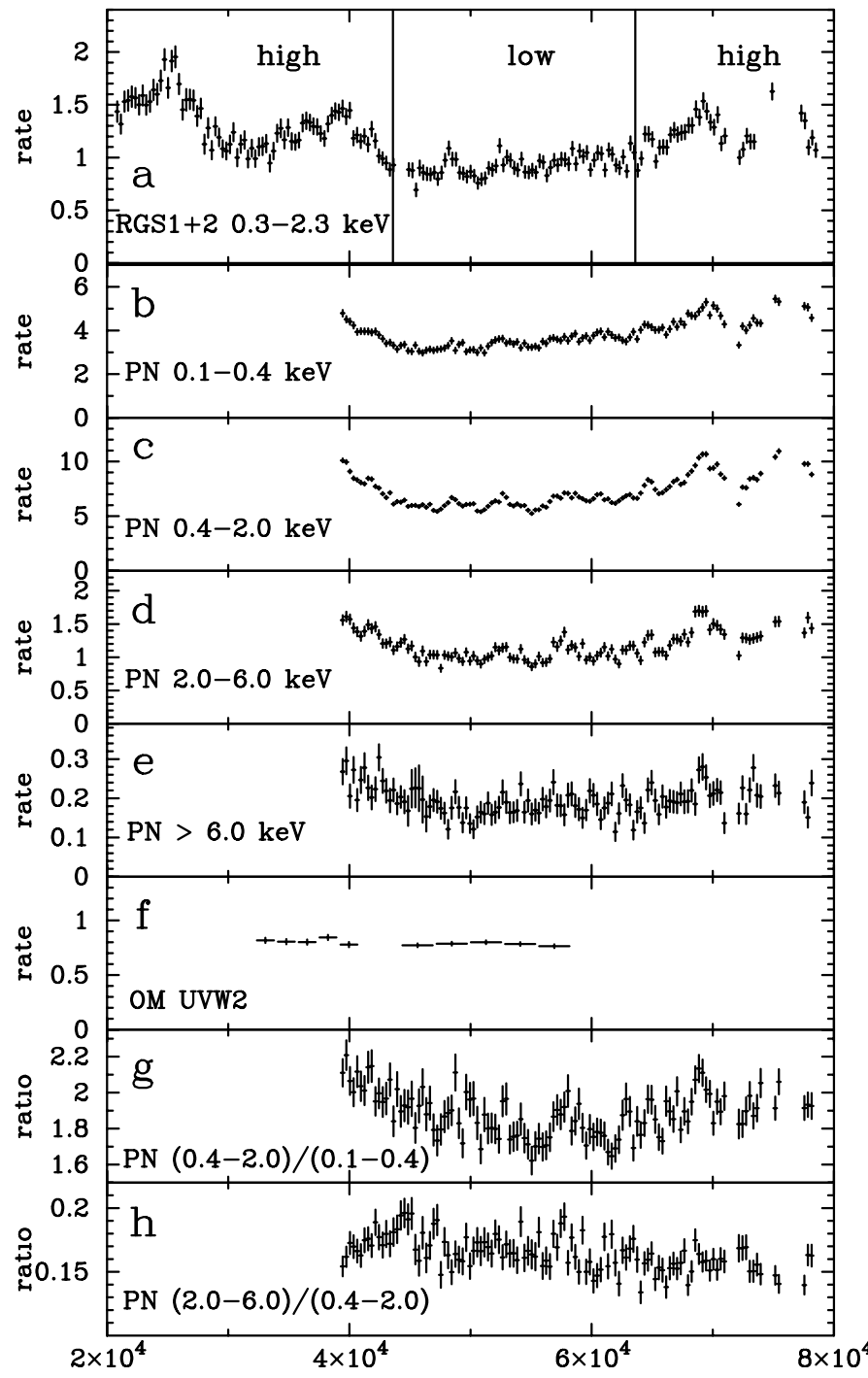

Time (s) from 2000-05-20.0

Fig. 4. Timeseries of Mrk 766 in the RGS a), EPIC PN b-e) and OM through the UVM2 filter f). The $y$ axis units for panels a-f) are count $\mathrm{s}^{-1}$. Panels $\mathbf{g}$ ) and $\mathbf{h}$ ) show hardness ratios constructed from the $\mathrm{PN}$ lightcurves, demonstrating that the source undergoes significant spectral variability during the observation

Figure $5 \mathrm{c}$ shows the ratio of the data to these best fit power law models. The two ratios are indistinguishable except below $0.7 \mathrm{keV}$, where the higher flux spectrum has a smaller fractional contribution from the soft excess emission. The overall spectral variability is therefore well characterised by an X-ray continuum which softens as it increases in flux combined with a less variable or constant component at low energy. This scenario would naturally explain the behaviour of the hardness ratios and why the $0.4-2.0 \mathrm{keV}$ lightcurve is the most variable of the PN lightcurves (Sect. 4). A variable warm absorber is not required to account for the spectral variability (as was suggested by Matt et al. 2000), because the deficit of photons between 0.7 and $2 \mathrm{keV}$ relative to the extrapolated higher

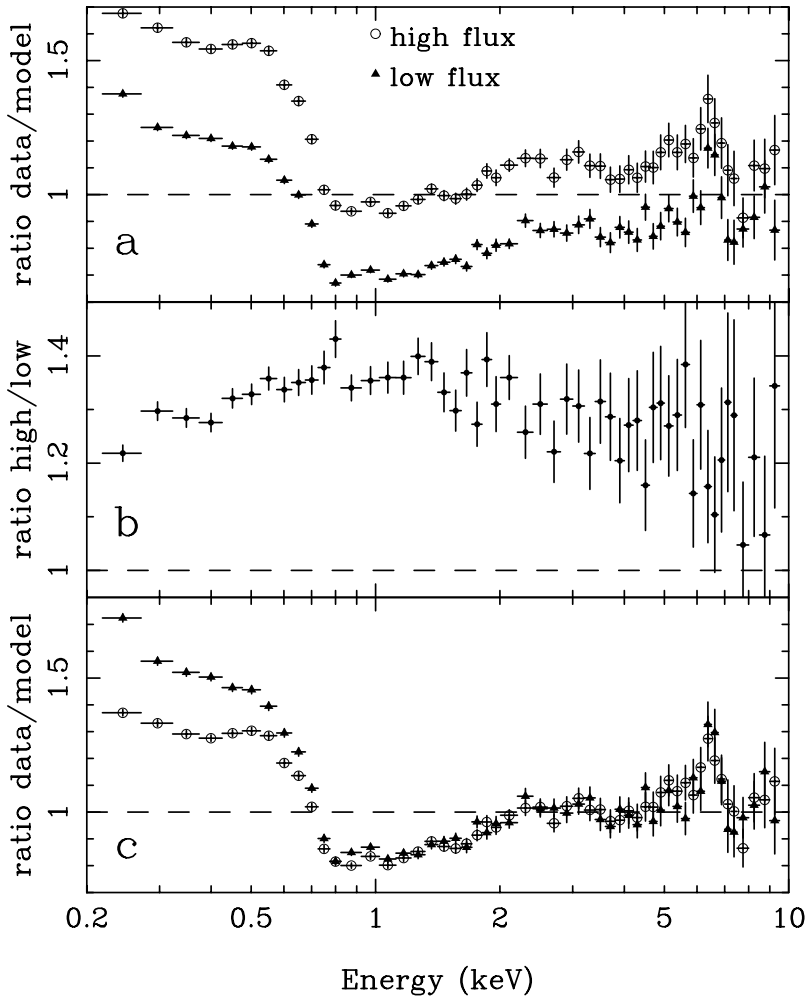

Fig. 5. a) Ratio of high flux and low flux spectra (see Sect. 5) to the same power law model. The open circles are used for the high flux spectrum and closed triangles for the low flux spectrum. b) Ratio of the high and low flux spectra. c) Ratio of high and low flux spectra to power law models fit individually to the two spectra, with symbols as in panel a)

energy power law spectrum is the same in the high and low flux spectra.

The spectral variability between 0.3 and $2 \mathrm{keV}$ can be examined at higher resolution using the RGS data. The high and low flux RGS1 spectra of Mrk 766, in 16 channel bins, are shown in Figs. 6a,b. Figure 6c shows the difference between these two spectra, and Fig. 6d shows the ratio of the high flux to low flux spectra. As is found for the PN spectra, the ratio of the high flux to low flux RGS spectra is smaller close to $0.3 \mathrm{keV}$ than above $1 \mathrm{keV}$. The $0.7 \mathrm{keV}$ edge-like feature is present in the low flux spectrum and the high flux spectrum. It also appears to be in the difference spectrum (though not the ratio spectrum) and hence if this feature is produced by a relativistic emission line, as proposed by Branduardi-Raymont et al. (2001), the line is varying with the continuum.

\section{Discussion}

The standard paradigm for the medium energy X-ray continuum emission in radio-quiet AGN is that it is due to Compton up-scattering of soft UV photons in some form of hot corona (Mushotzky et al. 1993 and references therein). The seed UV photons could be produced either by an accretion disk (Haardt et al. 1997) or from low temperature $\left(T \sim 310^{5} \mathrm{~K}\right)$ thermal emission in the corona itself 


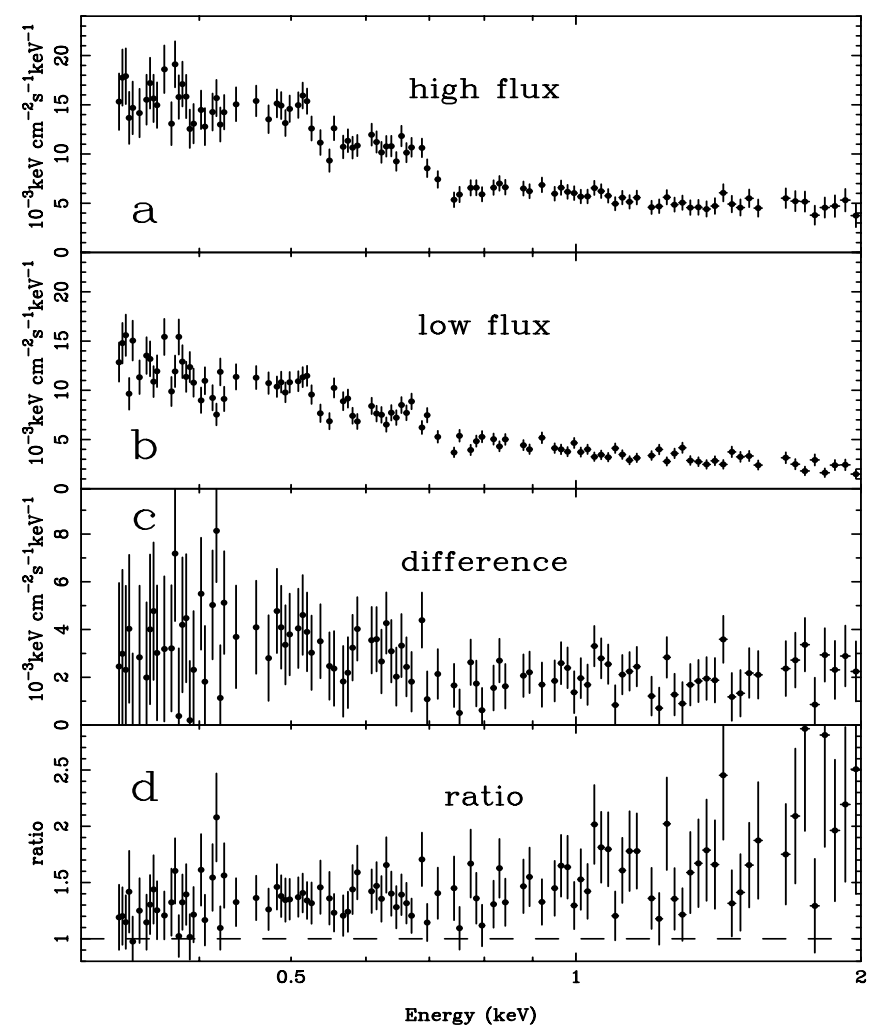

Fig. 6. a) High flux and b) low flux RGS1 spectra. c) Difference between the high and low flux spectra, d) ratio of the high flux spectrum to the low flux spectrum

(Torricelli-Ciamponi \& Courvoisier 1995). In Mrk 766 the broad Fe $\mathrm{K} \alpha$ line seen in the EPIC spectra provides strong evidence for the presence of a cool, optically thick accretion disk (Tanaka et al. 1995). This, and the lack of narrow coronal emission lines in the long wavelength part of the RGS spectra, favours the accretion disk as the source of the UV-soft X-ray bump in Mrk 766.

The UV-soft X-ray accretion disk emission is effectively bracketed by the soft $\mathrm{X}$-ray excess at $\lesssim 0.7 \mathrm{keV}$, and the UV emission observed in the OM. The behaviour of the $(0.4-2.0 \mathrm{keV}) /(0.1-0.4 \mathrm{keV})$ hardness ratio (Sect. 4), and the X-ray spectral variability (Sect. 5 ) implies that the soft excess varies significantly less than the Comptonised continuum. Furthermore, the OM lightcurves are consistent with zero variability in the UV. It therefore appears that the Compton upscattered X-ray continuum varies much more than its source of seed photons, the UV-soft X-ray bump. This implies that either the Comptonising corona is highly susceptible to changes in the soft photon field or that the variability of the Comptonised component is intrinsic to the corona itself and is unrelated to variations in the soft photon field. Something specific to the corona such as changing magnetic fields or the spectrum of particles injected into the corona would therefore provide a good explanation for the X-ray variability.

\section{Conclusions}

We have presented RGS, EPIC and OM spectra and lightcurves of Mrk 766 from the $60 \mathrm{ks} X M M-N e w t o n \mathrm{PV}$ phase observation. In all three EPIC cameras the spectra above $2 \mathrm{keV}$ are well described by a power law with a broad Fe $\mathrm{K} \alpha$ emission line. Relative to the power law, the EPIC spectra show a deficit of photons between $\sim 0.7$ and $2 \mathrm{keV}$ and excess emission at lower energies. The RGS spectra contain no strong, narrow emission lines. Warm absorber edges are not seen at the expected energies of OVII and OVIII in the RGS spectra; an edge-like feature at $0.7 \mathrm{keV}$ could only be due to OVII if the absorbing material were redshifted more than $10000 \mathrm{~km} \mathrm{~s}^{-1}$ relative to the system. Significant variability is detected in the X-ray lightcurves and hardness ratios, while no variability is detected in the UV with the OM. A variable warm absorber is not required to account for the spectral variability, which can be explained by a medium energy continuum component which softens as it increases in flux, with an additional contribution from a less variable emission component at low energy. Changes in magnetic fields threading the accretion disk corona, or the spectrum of particles injected into the corona, can explain the observed spectral variability.

\section{References}

Branduardi-Raymont, G., Sako, M., Kahn S. M., et al. 2001, A\&A, 365, L140

Fabian, A. C., Rees, M. J., Stella, L., \& White, N. E. 1989, MNRAS, 238, 729

Haardt, F., Maraschi, L., \& Ghisellini, G., 1997 ApJ, 476, 620

Kaastra, J. S., Mewe, R., Liedahl, D. A., Komossa, S., \& Brinkman, A. C. 2000, A\&A, 354, L83

Laor, A. 1991, ApJ, 376, 90

Leighly, K. M., Mushotzky, R. F., Yaqoob, T., Kunieda, H., \& Edelson, R. 1996, ApJ, 469, 147

Mathur, S. 2000, MNRAS, 314, L17

Matt, G., Perola, G. C., Fiore, F., et al. 2000, A\&A, in press [astro-ph/0009322]

Molendi, S., \& Maccacaro, T. 1994, A\&A, 291, 420

Mushotzky, R. F., Done, C., \& Pounds, K. A. 1993, ARA\&A, 31,717

Nandra, K., George, I. M., Mushotzky, R. F., Turner, T. J., \& Yaqoob, T. 1997, ApJ, 477, 602

Otani, C., Kii, T., Reynolds, C., et al. 1996, PASJ, 48, 211

Page, M. J., Carrera, F. J., Mittaz, J. P. D., \& Mason, K. O. 1999, MNRAS, 305, 775

Pounds, K. A., Nandra, K., Stewart, G. C., George, I. M., \& Fabian, A. C. 1990, Nat, 344, 132

Tanaka, Y., Nandra, K., Fabian, A. C., et al. 1995, Nat, 375, 659

Torricelli-Ciamponi, G., \& Courvoisier, T. J.-L. 1995, A\&A, 296,651

Walter, R., \& Fink, H. H. 1993, A\&A, 274, 105 